\title{
Explaining Dark Matter Without New Physics?
}

\author{
Stephane H. Maes ${ }^{1}$
}

June 21, 2020 (updated)

\begin{abstract}
:
In a multi-fold universe, gravity emerges from entanglement through the multi-fold mechanisms. As a result, gravity-like effects appear in between entangled particles or regions. When applied to astrophysics, these effects are analogous to additional matter within or around galaxies. This way, we recover behaviors that match expected and observed effects when dark matter would be present or missing. No New Physics is introduced in terms of new particles beyond the Standard Model or modifying long range gravity: only the modeling of gravity as emerging from entanglement, in a multi-fold universe.
\end{abstract}

\section{Introduction}

The new preprint [1] proposes contributions to several open problems in physics like the reconciliation of General Relativity (GR) with Quantum Physics, explaining the origin of gravity proposed as emerging from quantum (EPR Einstein Podolsky Rosen) entanglement between particles, detailing contributions to dark matter and dark energy and explaining other Standard Model mysteries without requiring New Physics beyond the Standard Model other than the addition of gravity to the Standard Model Lagrangian. All this is achieved in a multi-fold universe that may well model our real universe, which remains to be validated.

With the proposed model of [1], spacetime and Physics are modeled from Planck scales to quantum and macroscopic scales and semi classical approaches appear valid till very small scales. In [1], it is argued that spacetime is discrete, with a random walk-based fractal structure, fractional and noncommutative at, and above, Planck scales (with a 2-D behavior and Lorentz invariance preserved by random walks till the early moments of the universe). Spacetime results from past random walks of particles. Spacetime locations and particles can be modeled as microscopic blackholes (Schwarzschild for photons and spacetime coordinates, and metrics between Reisner Nordstrom [2] and Kerr Newman [3] for massive and possibly charged particles - the latter being possibly extremal). Although surprising, [1] recovers results consistent with other like [4], while also being able to justify the initial assumptions of black holes from the gravity or entanglement model. The resulting gravity model recovers General Relativity at larger scale, as a 4-D process, with massless gravity, but also with massive gravity components at very small scale that make gravity significant at these scales. Semi-classical models also work well till way smaller scales than usually expected.

In this paper, we remain at a high level of discussion of the analysis and references are generic for the subjects. It makes the points accessible to a wider audience and keeps the door open to further papers or discussions devoted to details of interest. Yet, it requires the reader to review [1], as we do not revisit here all the details of the multifold mechanism or reconstruction of spacetime. More targeted references for all the material discussed here are compiled in [1].

\section{Attractive Potential Between Entangled particles}

${ }^{1}$ shmaes.physics@gmail.com

Cite as: Stephane H Maes, (2020), "Explaining Dark Matter Without New Physics?", viXra:2007.0006, https://vixra.org/ abs/2007.0006, or https://shmaesphysics.wordpress.com/2020/06/21/explaining-dark-matter-without-new-physics/, June 21, 2020. 
The key proposal in [1] is a mechanism of multi-folds designed to address the EPR paradox. It is shown that, with such a mechanism, (EPR) entanglement creates an attractive potential between entangled particles that behaves like gravity.

When involving virtual particles emitted around a source of energy, we recover GR equations (and the Hilbert Einstein action) at classical (and semi-classical scales). At very small scales, there are additional contributions of massive virtual particles that generate additional contributions.

Entanglements between particles create additional contributions expected to behave like additional gravity contributions or fluctuations that we expect to see for example near macroscopically entangled material like superconductors [10].

The effective potentials can be seen as in $\propto \frac{1}{r^{2}}$ (or in $\frac{1}{r}$ when it can be integrated over a region (uncertainty region or bundle of entangled particles. For Gravity, the integration goes over $[r, \infty)$, for all the previous sent virtual pairs), where $r$ is the distance between particle and center of mass or source).

The effects due to entanglement are very small in general at macroscopic scales; yet, just like for gravity, they add up when considering the combined effect across a galaxy.

\section{The Dark matter problem}

It has been extensively shown that dark matter, i.e. matter that has mass or energy and interacts with other matter only (or mostly) through gravity (at least long range), is required to explain behavior of the universe, in particular the rotational velocities of most galaxies $[5,6]$. Without dark matter, they would disintegrate, considering the amount of normal matter observed or modeled. Dark matter is expected to constitute $85 \%$ of the total matter in the universe. Many models confirm its existence with good consistency across the methods used to estimate or validate its effects.

Today, however, Physics cannot account for, or explain, the origin of dark matter. Proposed tentative solutions (to explain or avoid dark matter) range from changes to gravity with for examples modifications of the long-range behavior of (newton) gravity (e.g. MOND), large scale massive gravity versions of GR, additional long range bulk spacetime entanglement effects ${ }^{2}$ in (entropic) gravity models, or proposing actual candidates for dark matter like black holes or particles most of the time new and associated to New Physics (see [5] for an overview).

Dark energy is another mysterious content of our universe [6,7]. [8] shows how the multi-fold mechanisms proposed in [1] can contribute to an explanation of the dark energy.

\section{EPR Entanglement in Multi-fold Universe: A Source for Dark Matter}

In [1], entangled EPR pairs create attractive gravity like potential in between them towards the center of mass of these particles (and variations for multi-partite, nonhierarchical, entanglement).

Virtual pairs emitted by energy or matter contribute to gravity with the model of [1]. Any other entanglement between particles, especially real particle entanglement, is not counted in conventional gravity. These entanglements appear as additional gravity contributions.

\footnotetext{
2 These notions, as proposed in [11,12], are fundamentally different effects from what is proposed in [1]. [1] considers effects between particles. Entropic bulk entanglement are postulated as statistical effects between spacetime regions. Of course, [1] may be an enabler or an explanation for such effect; or not. It does not really matter within the scope of this paper.
} 
Entanglement can be, as shown in figure 1:

- (1) Between particles emitted by stellar or other objects and these objects.

- (2) Between pairs of entangled particles moving in opposite directions.

- (3) Between surrounding matter or particles entangled with the above.

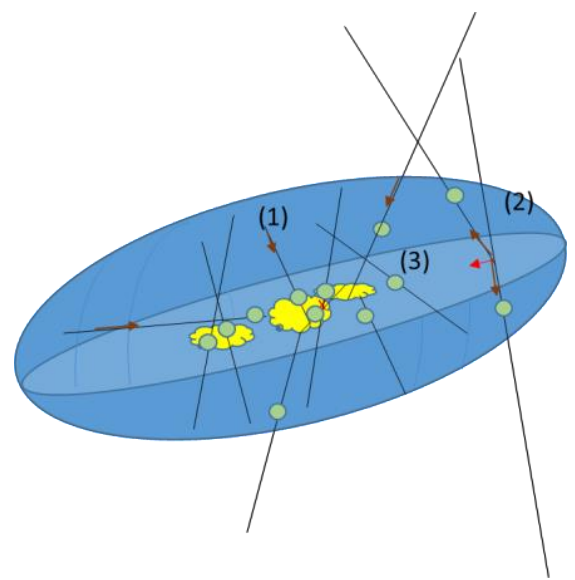

Figure 1: It illustrates how the different entanglements cases, discussed in the text, appear as dark matter with attraction towards the galaxy center and mass in the center or in halos. Green circles represent center of masses.

In all cases, the sources or centers of mass are located within the galaxy (especially in the center) and in surrounding halos. It matches the models for dark matter. The effect is a combination of cold and hot dark matter, but it always appear as cold matter. The dominant contributing particles involved in entanglement are photons and neutrinos. Of course, other cosmic radiations also contribute.

It is also well known that dark matter present some challenges for conventional explanations based on modified gravity or on particles because there are cases of galaxies where no or very little dark matter is inferred (See [9] for an example-more references can be found in [1]). It is hard to explain gravity laws or particles that would be sometimes be modified or sometimes be there; but not always.

It is not a problem with the multi-folds mechanism of [1].

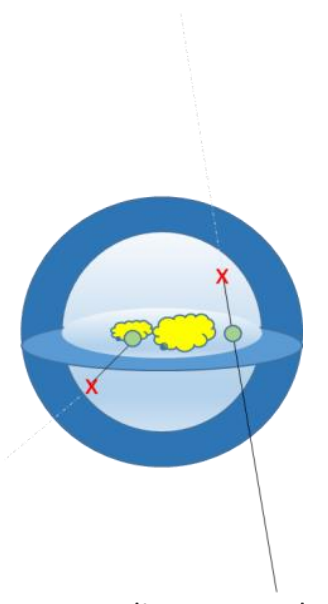

Figure 2: In globular cases, with enough matter surrounding, entanglement may be destroyed before it has the desired effects, therefore giving the impression of missing dark matter. 
In the model of [1], if matter is distributed (e.g. Globular galaxy - see Figure 2) in a way that intercept most particles early and disentangle them on their way out of a galaxy region, the effect weakens or disappears... It matches the few galaxy examples that miss dark matter.

This model and explanation is therefore able to account for dark matter, at least partially (till quantitatively estimated), and that is qualitatively consistent with observations; including when dark matter would be observed as missing.

The arguments in [1] are only qualitative, not yet quantitative. More work is needed to see if quantitative estimates make sense and may suffice to explain dark energy. Of course, other effects can also play along.

Also, this analysis is for a Multi-fold universe as in [1]. [1] details arguments and ways to check its relationship with the real universe. Besides properties that can be experimentally verified (in the future because of the macroscopic weakness of gravity and gravity like effects for entangled systems), [1] shows how the multi-fold mechanisms and behaviors are in many aspects in today's conventional physics, that, at times, anticipates the behaviors modeled of a multi-fold universe. In addition, [1] explains many results obtained in gravity, quantum mechanics, General Relativity, superstring theory, Loop Quantum Gravity and the AdS/CFT correspondence conjecture. All these works attempt to come up with models for the real universe. It is at least a good sign that [1] may provide an interesting model of the real universe.

Our proposal has no equivalent or variations for a non multi-fold universe: the source of dark matter effects come directly from the multi-folds mechanism as proposed in [1] and the resulting attraction towards the source or center of mass as a result of entanglement. Even other models, that link entanglement and gravity, may not help as the multi-fold universe do, as none have clearly identified such a gravity-like attraction as a result of entanglement. Any model where gravity appears between entangled particles could support the proposal from this paper.

\section{Conclusions}

We believe that [1] makes a compelling case for the consistency of its multi-fold proposal. The present paper shows how the mechanisms of multi-fold universes can help address the challenges with dark matter as well as the situation where it is believed to be missing.

Combined with [8], it is remarkable that the mechanism of [1] can contribute to effects like inflation, small cosmological constant and dark energy and now dark matter; that it be present or missing.

While steps in the right direction in terms of validating [1], future work should aim at providing quantitative estimates to further determine viability of the proposal or completeness of the explanation, versus just contributing to what happens, which would already be satisfying.

The proposed explanation of dark matter is also an attractive validation candidate for the proposal that entanglement generates gravity like contributions $[1,10]$.

References: (most references come from popular science to make the discussion more approachable)

[1]: Stephane H. Maes, (2020) "Quantum Gravity Emergence from Entanglement in a Multi-Fold

Universe", viXra:2006.0088v1, (June 9, 2020).

[2]: https://en.wikipedia.org/wiki/Reissner\%E2\%80\%93Nordstr\%C3\%B6m metric

[3]: https://en.wikipedia.org/wiki/Kerr-Newman metric

[4]: Burinskii, Alexander, (2008), "The Dirac-Kerr-Newman electron", arXiv:0507109v4 
[5]: https://en.wikipedia.org/wiki/Dark matter

[6]: B. Clegg (2019), "Dark Matter and Dark Energy: The Hidden 95\% of the Universe", Icon Books Ltd

[7]: https://en.wikipedia.org/wiki/Dark energy

[8]: Stephane H Maes, (2020), "Explaining Dark Energy, Small Cosmological Constant and Inflation Without New

Physics?", viXra:2006.0261v1, https://shmaesphysics.wordpress.com/2020/06/19/explaining-dark-energy-smallcosmological-constant-and-inflation-without-new-physics/, June 19, 2020.

[9]: Shany Danieli, Pieter van Dokkum, Charlie Conroy, Roberto Abraham, and Aaron J. Romanowsky, (2019), "Still Missing Dark Matter: KCWI High-resolution Stellar Kinematics of NGC1052-DF2", The Astrophysical Journal Letters, Volume 874 , Number 2

[10]: Stephane H Maes, (2020), "Entanglement Concretizes Time in a Multi-fold

Universe", https://shmaesphysics.wordpress.com/2020/06/28/entanglement-concretizes-time-in-a-multi-folduniverse/, June 28, 2020.

[11]: Erik P. Verlinde (2010), "On the Origin of Gravity and the Laws of Newton", arXiv:1001.0785

[12]: Erik Verlinde, (2016), "Emergent Gravity and the Dark Universe", arXiv:1611.02269v2 